\title{
Impact of Modified Vesicular Drug Delivery Systems on Polyherbal Formulations for Challenging Ailments
}

\author{
Sumathi $\mathrm{A}^{1 *}$, Suriyaprakash $\mathrm{TNK}^{1}$, Lakshmana Prabu $\mathrm{S}^{2}$ and \\ Balan $\mathbf{P}^{3}$ \\ ${ }^{1}$ Department of Pharmaceutics, Al Shifa College of Pharmacy, Perinthalmanna, \\ Kerala, India
}

\section{Review Article \\ Volume 1 Issue 2}

Received Date: October 13, 2017

Published Date: November 03, 2017

${ }^{2}$ Department of Pharmaceutical Technology, University College of Engineering (BIT Campus), Anna University, Tiruchirappalli, Tamilnadu, India

${ }^{3}$ Department of Pharmaceutical Chemistry, Al Shifa College of Pharmacy, Perinthalmanna, Kerala, India

*Corresponding author: Sumathi A, Department of Pharmaceutics, Al Shifa College of Pharmacy, Perinthalmanna, Kerala, India, E-mail: venisumathi@gmail.com

\section{Abstract}

Herbal plants have enormous therapeutic potential which should be explored through the fabrication these drugs in novel drug delivery systems. The major limiting factors viz. lipid solubility and molecular size of the phytoconstituents to pass the biological membrane can be overcome by the usage of novel carriers. Also, the concept of multi-targeted therapy exists in traditional system of medicine. These multi-components existing in the extracts of natural products act simultaneously on multiple targets. Thus, polyherbal therapies involve the combination of various types of agents from different plant sources and thereby used to enhance therapeutic efficacy. They also minimize the risk of development of drug resistance. Standardized plant extracts when administered through novel drug delivery system show much better pharmacodynamic and pharmacokinetic profile. Hence more amount of active constituent becomes available at the site of action at same or lesser dose compared to the conventional plant extract. Hence, the therapeutic action becomes enhanced, more detectable and prolonged. Several excellent phytoconstituents have been successfully delivered using NDDS (Novel Drug Delivery System). Thus, this review is focused on the potential of phytosomes, a modified vesicular drug delivery system in the development of plant actives and extracts.

Keywords: Herbal plant; Polyherbal; NDDS; Phytoconstituents; Phytosomes

\section{Introduction}

Herbal formulations, a type of dosage form has been emerging in the field of medicine and started dwelling a unique consign in the international market. It consists of one or more herbs or processed herb in specified quantities to provide specific benefits meant for diagnosis, treatment, mitigation or alteration of physiology or structure of human beings or animals. It has also meant for nutritional or supplementary or cosmetic or other benefits. Either the whole plants or plant parts or fragments are subjected to an assortment of treatments such as extraction, distillation, expression, fractionation, purification, concentration or fermentation to acquire herbal preparations [1-3]. All the way through herbal source, there is an escalating interest in identifying retorts for challenging ailments and its applications in modern medicine. Despite having 
excellent bioactivity in vitro, several plant extracts lack a significant in vivo correlation due to their poor lipid solubility or improper molecular size which in turn results poor absorption and/or poor bioavailability. Novel drug delivery systems for herbal drugs have an impending outlook for enhancing the therapeutic activity and also to overcome the tribulations associated with herbal medicines [4-6].

\section{Role of Carrier Systems in Delivering Phytoconstituents}

Standardized plant extracts viz. flavonoids, terpenoids, tannins, xanthones, etc., when fabricated through novel drug delivery system show much better profile in terms of absorption, bioavailability and therapeutic activity. Therefore, the amount of active phytoconstituents available at the site of action (liver, brain, heart, kidney, etc.) is high compared to conventional plant extract. Later, it was found that complexation with certain other substances like phospholipids substantially improved the bioavailability of such extracts by enhancing the absorption of combined phytoconstituents [7-9]. Today, in the midst of emerging drug delivery systems, phospholipid based carriers are of much importance because of intense advantages associated with them. Phospholipids are complex molecules that are used in all known life forms to make cell membranes. They are building blocks for cell membrane, making up the matrix into which fit a large variety of proteins that are enzymes, transport proteins, receptors, and other biological energy converters. In humans and other higher animals, the phospholipids are also employed as natural digestive aids and as carriers for both fatmiscible and water miscible nutrients. The various phospholipid based drug delivery carriers include phytosomes, liposomes, long circulating liposomes, ethosomes, transferosomes and nanochelates, etc, $[10,11]$.

\section{Phytosomes for Polyherbal Formulations}

Phytosome, a type of phospholipid based carrier system is a patented technology developed by a leading manufacturer of drugs and nutraceuticals. It is used to incorporate standardized plant extracts or water soluble phytoconsituents into phospholipids at molecular level. Thus, lipid compatible molecular complexes are formed called as phytosomes. They are the most advanced forms of herbal extracts that exhibits better pharmacokinetic and pharmacodynamic profile than conventional herbal extracts. They are also defined as a type of vesicular drug delivery system or modified vesicular drug delivery system of liposomes. It is a complex of a phytoconstituents and a phospholipid having a little cell like structure ("phyto" means plant; "some" means cell-like) [12-14].

\section{Composition of Phytosomes}

Phytosomes constitute a bioactive phytomolecule and a phospholipid. The phospholipid molecular structure includes a water soluble head and two fatsoluble tails. Because of this dual solubility, the phospholipid acts as an effective emulsifier and notable that it is one of the chief components of the membranes in our cells. These properties enable them to deliver the active moieties to the desired site or location of the body which have been already reported. Physicochemical and spectroscopical studies revealed the novelty of these entities [15-17].

Most of the bioactive constituents of phytomedicines are water-soluble compounds like flavonoids, glycosides and terpenoids. Among them, flavonoids are a major class of bioactive compounds possesses broad therapeutic spectrum of activities. Because of hydrophilic head and lipophilic outer layer, phytosomes shows better absorption and thereby produce better bioavailability than the conventional herbal extracts containing phytomedicines [18].

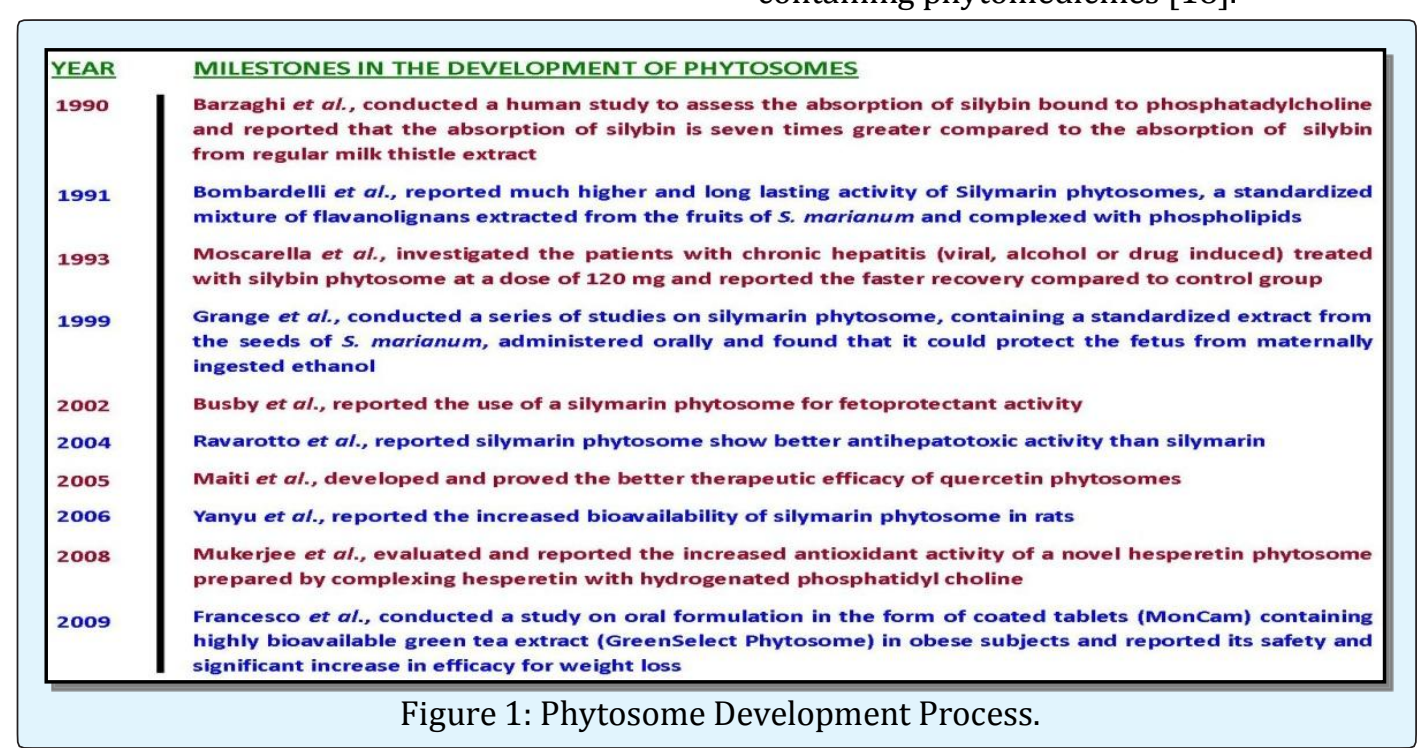




\section{Bioequivalence \& Bioavailability International Journal}

\section{Preparation of Phytosomes}

The phytoconstituents of herbal extracts lend themselves for the binding to phospholipids directly. Thus, phytosomes result from the reaction of a stoichiometric amount of the phospholipid with the standardized extract or polyphenolic constituents in a non polar solvent. Phospholipids are bifunctional compounds and hence, the phytoconstituents produce a lipid compatible molecular complex with phospholipids, also called as phyto-phospholipid complex.

The bioactives or phytomolecules are anchored through chemical bonds to the polar head of the phospholipids. This commotion can be demonstrated by specific spectroscopic techniques. The precise chemical analysis indicates that the unit phytosome is usually a flavonoid or any other phytomolecule linked with at least one phospholipid molecule. The phytosome technology produces a little micro cell, whereby the plant extract or its active constituent is protected from destruction of gastric secretions and gut bacteria owing to the gastroprotective property of phospholipids [1921].

\section{Phytosome Technology}

In the phytosome technology, the novel complexes are prepared by reacting one mole (rarely 2-3 moles) of a natural or synthetic phospholipid with one mole of a phytoconstituent either alone or in the natural mixture in aprotic solvent. In the complex formation, the ratio between these two moieties ranges from 0.5-2.0 moles. The formed complex can be isolated by precipitation with non solvent such as aliphatic hydrocarbons or by lyophilization or by spray drying. Phospholipids are selected from the group which includes phosphatidic acid, phosphatidyl choline, phosphatidyl ethanolamine, phosphatidyl serine in which acyl group may be same or different and derived from palmitic, stearic, oleic and linoleic acid. Selection of flavonoids are done from the group consisting of quercetin, kaempferol, quercretin-3, rhamnoglucoside, quercetin-3-rhamnoside, hyperoside, vitexine, diosmine, 3- rhamnoside, (+) catechin, (-) epicatechin, apigenin-7-glucoside, luteolin, luteolinglucoside, ginkgonetine, isoginkgonetine and bilobetine. Few liposomal drugs complex operate in the presence of the water or buffer solution whereas phytosomes operate with the solvent having a reduced dielectric constant. Flavonoids, which are insoluble in chloroform, diethyl ether or benzene, become extremely soluble in these solvents after forming phytosomes. Change in physic-chemical property is due to the formation of a true stable complex, phytosome [22-24].

\section{Advantages of Phytosomes}

- Enhances the absorption of lipid insoluble polar phytoconstituents through oral as well as topical route.
- Showing better bioavailability.

- Significantly greater therapeutic benefit.

- Reduced dose because of improved absorption of active constituent(s).

- Better stability profile due to the formation of chemical bonds between phospholipid molecules and phytoconstituents.

- Protects the bioactives/phytoconstituents from destruction by digestive secretions and gut bacteria.

- Able to execute the transition from a hydrophilic environment into the lipophilic environment of the cell membrane and from there into the cell finally reaching the blood.

- Exhibits better pharmacokinetic and pharmacodynamic profile than conventional herbal extracts [25-26].

\section{Limitations of Phytosomes}

Phytosome is a complex unit of phospholipid and phytoconstituent which specifically bonded together in 1:1 or $2: 1$ ratio depending on its characteristic features. Spectroscopic studies revealed that the phospholipids-substrate interaction is due to the formation of hydrogen bonds between the polar head of phospholipids and the polar functionalities of the substrate. Thus, it can able to produce better absorption and increased bioavailability at the site of action. But, the phytoconstituents are rapidly eliminated from phytosomes which might decrease their duration of action [27].

\section{Techniques used for the Evaluation of Phytosomes}

- Visualization of phytosomes can be achieved using Transmission Electron Microscopy (TEM) and by Scanning Electron Microscopy (SEM).

- Vesicle Size and Zeta Potential can be determined by Dynamic Light Scattering (DLS) using a computerized inspection system and Photon Correlation Spectroscopy (PCS).

- Entrapment Efficiency of a drug by phytosomes can be measured by the ultracentrifugation technique.

- Transition Temperature of the vesicular lipid systems can be determined by differential scanning calorimetry.

- Surface tension activity measurement of the drug in aqueous solution can be measured by the ring method in a Du Nouy ring tensiometer.

- Vesicle stability can be determined by assessing the size and structure of the vesicles over time. The mean size is measured by DLS and structural changes are monitored by TEM.

- Drug content can be quantified by a modified high performance liquid chromatographic method or by a suitable spectroscopic method. 
- Spectroscopic evaluations can be done to confirm the formation of a complex or to study the reciprocal interaction between the phytoconstituents and the phospholipids through ${ }^{1} \mathrm{H}-\mathrm{NMR},{ }^{13} \mathrm{C}-\mathrm{NMR}$ and FTIR spectroscopic methods.

- In vitro and In vivo evaluations are done based on the expected therapeutic activity of the biologically active phytoconstituents present in the phytosomes. For example, in vitro anti-hepatotoxic activity can be assessed by antioxidant and free radical scavenging activity of the phytosomes whereas in vivo antihepatotoxic activity can be assessed by studying the effect on animals against thioacetamide-paracetamol or alcohol-induced hepatotoxicity [28-31].

\section{Applications of Phytosomes}

Phytosome technology has been effectively used to enhance the bioavailability of many herbal extracts including Ginkgo biloba extract, Silybum marianum extract, Curcumin longum extract and Camellia sinensis extract (green tea). These phytosome vesicles deliver therapeutic compounds for a wide range of possible applications. It can be industrialized for various therapeutic uses, cosmetic applications and dietary supplements [32-50].

\begin{tabular}{|c|c|c|}
\hline Phytosome Formulations & Active Constituents & Pharmacological Activity \\
\hline Ginkgo biloba Phytosome & Flavonoids & $\begin{array}{c}\text { Cardio-protective, Hepato-protective and } \\
\text { Antioxidant }\end{array}$ \\
\hline Bilberry Phytosomes & Anthocyanosides & $\begin{array}{c}\text { Potent antioxidant, Improve capillary tone and } \\
\text { Reduce abnormal blood vessel permeability }\end{array}$ \\
\hline Ginseng Phytosome & Ginsenosides & Nutraceutical \\
\hline Green tea Phytosome & Epigallocatechin & Vein and Skin disorders \\
\hline Centella Phytosome & Terpenes & $\begin{array}{c}\text { Nutraceutical and Immunomodulator } \\
\text { folia }\end{array}$ \\
\hline Echinacea Phytosome & $\begin{array}{c}\text { Echinacosides from Echinacea august } \\
\text { Anticancer }\end{array}$ \\
\hline Ginseng Phytosome & Ginsenosides from Panax ginseng & Nutraceutical and Immunomodulator \\
\hline $\begin{array}{c}\text { Grape Seed Phytosome } \\
\text { Quercetin Phytosome }\end{array}$ & Procyanidins from Vitis vinifera & $\begin{array}{c}\text { Nutraceutical, Antioxidant and Cardio-protective } \\
\text { Antihypertensive }\end{array}$ \\
\hline Hawthorn Phytosome & Flavonoids from Crataegus sp. & $\begin{array}{c}\text { Nutraceutical, Cardio-protective and } \\
\text { hyperlipidemic }\end{array}$ \\
\hline $\begin{array}{c}\text { Olive oil Phytosome } \\
\text { Purcumin Phytosome }\end{array}$ & Poly phenols from Olea europaea oil & $\begin{array}{c}\text { Antioxidant, Anti inflammatory and Anti } \\
\text { Anticancer }\end{array}$ \\
\hline Palmetto berries & Fatty acids, alcohols and sterols & Treatment of Non-cancerous prostate enlargement \\
\hline
\end{tabular}

Table 1: Applications of Phytosomes.

\section{Future Scope}

The thorough literature survey divulges the different products of phytosomes and also demonstrated their significant therapeutic as well as health promoting properties as compared to conventional forms of plant extracts. Crude, partially purified or fractionated plant extracts can be standardized and then formulated as phytosomes for systematic investigation to expose any improved potential applications of the same. Thus, in future, phytosomes can be developed for different therapeutic purposes viz. hepatoprotective, cardiovascular disorders, liver diseases, antiinflammatory, Immunomodulator, anticancer, anti diabetic and also for prophylactic and health purposes as nutraceuticals.

\section{Conclusion}

Phytosomes, an emerging technology in drug delivery is being applied to phyto-pharmaceuticals for the improvement of bioavailability of herbal extracts. They are referred as advanced and novel form of botanicals or phytoconstituents. They have improved pharmacokinetics, pharmacodynamic and pharmacological parameters. Also, they have been reported to enclose wide scope in the field of cosmetics. The methods used for preparing phytosomes are simple and reproducible. Apart from that the phospholipids used in formulating phytosomes have their own beneficial effect to the body. Thus, the phytosome technology forms a bond between the phytoconstituents and novel drug delivery systems. 


\section{Bioequivalence \& Bioavailability International Journal}

\section{References}

1. Talalay P (2001) The importance of using scientific principles in the development of medicinal agents from plants. Acad Med 76(3): 238-247.

2. Heinrich M (2003) Ethnobotany and natural products: the search for new molecules, new treatments of old diseases or a better understanding of indigenous cultures. Curr Top Med Chem 3(2): 141-154.

3. Schmidt B, Ribnicky DM, Poulev A, Logendra S, Cefalu WT, et al. (2008) A natural history of botanical therapeutics. Metabolism 57(7 S 1): S3-9.

4. Vyas SP, Khar RK (2002) Targeted and Controlled Drug Delivery Novel Carrier Systems. $1^{\text {st }}$ edition, CBS Publishers, New Delhi Pp: 38-46.

5. Yie W Chien (1992) Novel Drug Delivery Systems. $2^{\text {nd }}$ edition, Marcel Dekker, New York Pp: 1-38.

6. Bertrand N, Leroux JC (2011) The journey of a drug carrier in the body: an anatomo-physiological perspective. Journal of Controlled Release 161(2): 152-163.

7. Agnihotri SA, Mallikarjuna NN, Aminabhavi TM (2004) Recent advances on chitosan-based micro and nanoparticles in drug delivery. Journal of Controlled Release 100(1): 5-28.

8. Sood A, Panchagnula R (2000) Peroral route: An opportunity for protein and peptide drug delivery. Chemical Reviews 101(11): 3275-3303.

9. Vasir JK, Tambwekar K, Garg S (2003) Bioadhesive microspheres as a controlled drug delivery system. Int. J. Pharm 255(1-2): 13-32.

10. Chan (2007) WCW: In: Bio-Applications of Nanoparticles. Landes Bioscience, Austin, TX, USA

11. Ziaie B, Baldi A, Lie M, Gu Y, Siegel RA (2004) Hard and soft micro machining for Biomems. Review of techniques \& examples of applications in microfluids and drug delivery. Advanced Drug Delivery Reviews 56(2): 145-172.

12. Bombardelli E, Curri SB, Della RL, Del NP, Tubaro A, et al. (1989) Complexes between phospholipids and vegetal derivatives of biological interest. Fitoterapia 60: 1-9.

13. Choubey A (2011) Phytosome: A Novel approach for Herbal Drug Delivery. International Journal of Pharmaceutical Sciences and Research 2(4): 807815.
14. Nilesh Jain BP (2010) Phytosome: A Novel Drug Delivery System for Herbal Medicine. International Journal of Pharmaceutical Sciences and Drug Research 2(4): 224-228.

15. Joseph A Kareparamban, Pravin H Nikam (2012) Phytosomes: A Novel Revolution in Herbal Drugs. International Journal of Research in Pharmacy and and Chemistry 2(2): 299-310.

16. Kidd P, Head K (2005) A review of the bioavailability and clinical efficacy of milk thistle phytosomes: a Silybin phosphatidylcholine complex. Alter Med Rev 10(3): 193-203.

17. Mauri P, Simonetti $P$, Gardana C, Minoggio $M$, Morazzoni P, et al. (2001) Liquid chromatography/ atmospheric pressure chemical ionization mass spectrometry of terpene lactones in plasma of volunteers dosed with Ginkgo biloba L. extracts. Rapid Commun Mass Spectrom 15(12): 929-934.

18. Rossi R, Basilico F, Rossoni G, Riva A, Morazzoni P, et al. (2009) Liquid chromatography/atmospheric pressure chemical ionization ion trap mass spectrometry of bilobalide in plasma and brain of rats after oral administration of its phospholipidic complex. J Pharm Biomed Anal 50(2): 224-227.

19. Bombardelli E (1991) Phytosome: new cosmetic delivery system. Boll Chim Farm 130(11): 431-438.

20. Bombardelli E, Cristoni A, Morazzoni P (1994) Phytosomes in Functional Cosmetics. Fitoterapia. 95: 387-401.

21. Bombardelli E, Spelta M, Loggia Della R, Sosa S, Tubaro A (1991) Aging Skin: Protective effect of silymarin -phytosomes. Fitoterapia 62(2): 115-122.

22. Tawheed Amin, Suman Vikas Bhat (2012) A review on phytosome technology as a novel approach to improve the bioavailability of nutraceuticals. International Journal of Advancements in Research \& Technology 1(3): 1-15.

23. Bombardelli E, Curri SB, Della RL, Del NP, Tubaro A, et al. (1989) Complexes Between Phospholipids and Vegetal Derivatives of Biological Interest. Fitoterapia 60: 1-9.

24. Mascarella S (1993) Therapeutic and Antilipoperoxidant Effects of SilybinPhosphatidylcholine Complex in Chronic Liver Disease, Preliminary Results. Curr Ther Res 53(1): 98-102.

25. Bombardelli E, Spelta M (1991) PhospholipidPolyphenol Complexes: A New Concept in Skin Care Ingredients. Cosm \& Toil 106: 69-76. 
26. Sharma S, Sikarwar M (2005) Phytosome: a review. Planta Indica 1(2): 1-3.

27. Sravanthi M, Shiva Krishna J (2013) Phytosomes: A Novel Drug Delivery for Herbal Extracts. Int. J. of Pharmaceutical Sciences and Research 4(3): 949959.

28. Amin T, Bhat SV (2012) A review on Phytosome Technology as a Novel Approach to improve the Bioavailability of Nutraceuticals. Int. J. Adv. Res. Technol 1(3): 1-5.

29. Kattamanchi G, Kalakonda SN, Battu GR (2017) Phospholipid Complex Technique for Superior Bioavailability of Phytoconstituents. Adv Pharm Bull 7(1): 35-42.

30. Busby A, LaGrange L, Edwards J, King J (2002) The use of A Silymarin/Phospholipid Compound As A Fetoprotectant From Ethanol-Induced Behavioral Deficits. J Herb Pharmacother 2(1): 39-47.

31. Francesco D P, Anna B M, Angela B, Maurizio L, Andrea C (2009) Green Select Phytosome as an Adjunct to a Low-Calorie Diet for Treatment of Obesity: A Clinical Trial. Altern Med Rev 14(2): 154160.

32. Maiti K, Mukherjee K, Gantait A, Ahamed HN, Saha BP, Mukherjee PK (2005) Enhanced Therapeutic Benefit of Quercetin-Phospholipid Complex In Carbon Tetrachloride Induced Acute Liver Injury In Rats: A Comparative Study. Iran J Pharmacol Ther 4(2): 84-90.

33. Maiti K, Mukherjee K, Gantait A, Saha BP, Mukherjee PK (2006) Enhanced therapeutic potential of naringenin-phospholipid complex in rats. J Pharm Pharmacol 58(9): 1227-1233.

34. Maiti K, Mukherjee K, Gantait A, Saha B, Mukherjee PK (2007) Curcumin-Phospholipid Complex: Preparation, therapeutic evaluation and pharmacokinetics study in rats. Int J Pharm 330(12): 155-163.

35. Mukherjee K, Maiti K, Venkatesh M, Mukherjee PK (2008) Phytosome of Hesperetin, A Value Added Formulation with Phytomolecules. 60 th Indian Pharmaceutical Congress, New Delhi Pp: 287.

36. Tedesco D, Steidler S, Galletti S, Tameni M Ravarotto L et al. (2004) Eficacy of SilymarinPhospholipid Complex in Reducing the Toxicity of Aflatoxin B1 in Broiler Chicks. Poult Sci 83(11): 1839-1843.

37. Yanyu X, Yunmei S, Zhipeng C, Quineng P (2006) The Preparation of Silybin-Phospholipid Complex and the Study on Its Pharmacokinetics in Rats. Int J Pharm 307(1): 77-82.

38. Morazzoni P, Montalbetti A, Malandrino S, Pifferi G (1993) Comparative pharmacokinetics of silipide and silymarin in rats. Eur J Drug Metab Pharmacokinet 18(3): 289-297.

39. Marena C, Ampertico P (1991) Preliminary clinical development of silepide, a new complex of in Silybin in toxic liver disorders. Planta Med 57(S2): A124-5.

40. Marczylo TH, Verschoyle RD, Cooke DN, Morazzoni P, Steward WP, et al. (2007) Comparison of systemic availability of curcumin with that of curcumin formulated with phosphatidylcholine. Cancer Chemother Pharmacol 60(2): 171-177.

41. Belcaro G, Cesarone MR. Dugall M, Pellegrini L, Ledda A, et al. (2010) Product-evaluation registry of Meriva $\AA$, a curcumin-phosphadidylcholine complex, for the complementary management of osteoarthritis. Planta Med. 52(2S1): 55-62.

42. Citernesi U, Sciacchitano M (1995) Phospholipids/active ingredient complexes. Cosm \& Toil 110(11): 57-68.

43. Barzaghi N, Crema F, Gatti G, Pifferi G, Perucca E (1990) Pharmacokinetic studies on Idb 1016, a silybin phosphatidylcholine complex in healthy human subjects. Eur J Drug Metab Pharmacokinet 15(4): 333-338.

44. Moscarella S (1993) Therapeutic and antilipoperoxidant effects of silybin phosphatidyl choline complex in chronic liver disease: Preliminary results. Curr Ther Res 53(1): 98-102.

45. La Grange L, Wang M, Watkins R, Ortiz D, Sanchez ME (1999) Protective effects of the flavonoids mixture, silymarin, on fetal rat brain and liver. J. Ethnopharmacol 65(1): 53-61.

46. Pietta P, Simonetti P, Gardana C, Brusamolino A Morazzoni P, et al. (1998) Relationship between rate and extent of catechin absorption and plasma antioxidant status. Biochem Mol Biol Int 46(5): 895903.

47. Magistretti Maria Jose, Bombardelli Ezio. 1987; U.S. Patent No-EP0209037. Pharmaceutical compositions containing flavanolignans and phospholipida active principles.

48. Kidd PM (1996) Phosphatidyl choline: a superior protectant against liver disease. Altern Med Rev 1(4): 258-274 


\section{Bioequivalence \& Bioavailability International Journal}

49. Valenzuela A, Aspillaga M, Vial S, Guerra R (1989) Selectivity of silymarin on the increase of the glutathione content in different tissues of the rat. Planta Med 55(5): 420-422.
50. Hiking H, Kiso Y, Wagner H, Fiebig M (1984) Antihepatotoxic actions of flavonolignans from Silybum marianum fruits. Planta Med 50(3): 248250. 